\title{
Availability of Essential Commodities and Related Bottlenecks for Community Health System: Systematic Literature Review
}

Ami Pradhan ( $\square$ amipradhan@gmail.com )

UNICEF https://orcid.org/0000-0002-9961-4322

Erin Bogue

UNICEF USA

Benjamin Schreiber

UNICEF

Hannah Sarah Dini

UNICEF

Hitesh Hurkchand

UNICEF

Jane Briggs

Management Sciences for Health (MSH)

Madeleine Ballard

Community Health Impact Coalition

Research article

Keywords: Community Health Workers, stock-out, pharmaceutical services, supply chain, health system strengthening, product availability, community health

Posted Date: August 22nd, 2020

DOI: https://doi.org/10.21203/rs.3.rs-24276/v2

License: (c) (1) This work is licensed under a Creative Commons Attribution 4.0 International License. Read Full License 


\section{Abstract}

Background: This paper explores the extent of community-level stock-out of essential and program commodities for Maternal, Newborn and Child Health (MNCH) among Community Health Workers (CHWs) in Low- and Middle-Income Countries (LMICs), and identifies the underlying bottlenecks leading to such stock-outs.

Methods: A systematic literature review was conducted of published and grey literature. 48 studies containing information on the number or percentage of CHWs or health facilities (HFs) stocked-out, or reasons for stock-outs at these levels, were included. In addition, interviews were conducted with domain experts from different organizations working on community health to identify primary reasons for stockouts. The qualitative data regarding reasons for stock-outs were categorized under different themes, using a content analysis method.

Results: $48.09 \%$ [Cl 95\%: 39.28 - 56.90] of the Community Health Workers and $54.76 \%$ [Cl 95\%: 43.54 65.98] of the health facilities in SSA countries included in this study experienced stock-outs of essential commodities. A hypothesis test showed no significant difference in stock-out rates between $\mathrm{CHWs}$ and HFs. The most frequently cited reason for CHW stock-outs was a lack of financial resources, leading to inadequate national level stocks, affecting supply available at the last mile. Moreover, issues at HF and CHW levels in the following areas contributed to stock-outs: transportation, data and estimation of needs, human resources, and stock management and storage. These significant bottlenecks hinder the ability of CHWs to save lives.

Conclusion: Stock-outs of health commodities impact almost half of CHWs, preventing effective service delivery. Many factors contribute to stock-outs, which occur at all levels of the health supply chain. A system strengthening approach is necessary to reduce $\mathrm{CHW}$ stock-outs. Sparse data, differing definitions of stock-outs, and inconsistent reporting metrics posed significant challenges to analyzing results from reviewed studies. Therefore, a set of standard metrics to measure the rate, period, and frequency of stockouts in future studies is recommended.

\section{Background}

Community Health Workers (CHWs) are paraprofessionals or lay individuals based in the community who provide health services to both urban and rural communities. ${ }^{[i]}$ The role of $\mathrm{CHWs}$ is increasingly recognized as a significant step towards achieving Universal Health Coverage and the post-2015 health agenda ${ }^{[i i]} \mathrm{CHW}$ cadres vary across countries and have different names, levels of training, responsibilities and professional status. ${ }^{[i i i]}$ They also provide different packages of care in different countries. CHWs play a crucial role in providing (a) access to primary health care services through counselling, and (b) preventive and routine curative health services to families who previously had limited contact with trained health workers ${ }^{[i v]}$ in LMICs. ${ }^{[v],[v i]}$ 
CHWs significantly reduce MNCH morbidities and mortalities ${ }^{[\text {Viil] }[\text { viil] }}$ through distribution and appropriate utilization of health products, however, this effect was only observed if $\mathrm{CHWs}$ had continuous access to essential supplies. ${ }^{[i x]} \mathrm{CHW}$ performance is greatly influenced by the resources available to these workers. ${ }^{[x]}$ If $\mathrm{CHWs}$ are properly trained and equipped, they can reduce child deaths from malaria, pneumonia, and diarrhea by up to $60 \%$ through the delivery of Integrated Community Case Management (iCCM). ${ }^{[x]}$ Systematic and narrative reviews have found that stock-out of health commodities is a major hindrance to $\mathrm{CHW}$ productivity and motivation. ${ }^{[x i] \mid x i i]]}$

Understanding the extent and causes of stock-outs at the community level is critical for optimizing $\mathrm{CHW}$ programs. ${ }^{[x i]]}$ Some projects, such as Improving Supply Chains for Community Case Management of Pneumonia and Other Common Diseases of Childhood (SC4CCM), implemented by JSI Research \& Training Institute, have documented stock-outs of health commodities at community level in several countries. However, there is a lack of systematic literature review documenting the extent of CHW stockouts and elucidating underlying bottlenecks to product availability at community level. This paper, therefore, is trying to find out what extent of the CHWs lacks essential commodities to render service efficiently and what are the reasons for such stock outs

\section{Methods}

This systematic literature review documents the extent of stock-outs at $\mathrm{CHW}$ and $\mathrm{HF}$ levels for $\mathrm{MNCH}$ commodities in LMICs and describes the underlying bottlenecks that cause these stock-outs.

We employed two methods: a) a review of published and grey literature on extent of and causes of stockouts at $\mathrm{CHW}$ and $\mathrm{HF}$ levels; and b) interviews with 30 domain experts, identified through snowball method. Stock-outs at HFs were included, as in most settings these facilities are responsible for resupplying products to CHWs. A two-sample test for proportions was applied to determine whether a statistical link existed between the CHW and HF stock-out rates.

\subsection{Search Strategy}

During August and September 2018, we reviewed published and unpublished articles in four electronic databases: 1) PubMed, 2) Global Health via Ovid, 3) Web of Science, 4) Embase via Ovid. In addition, we searched the Google and Google Scholar search engines, and The World Health Organization's website for relevant articles during the same period (see Supplementary File, Appendix 1).

A team of supply chain (SC) experts was engaged to solicit evidence-based, unpublished literature and to identify contact persons in various organizations working with CHWs in LMICs. Grey literature was accessed by requesting documents and reports from identified contact persons in various organizations (e.g. USAID, WHO, VillageReach, Save the Children, JSI, MSH, Last Mile Health, World Vision, etc.). Different platforms, such as the Interagency Supply Chain Group (ISG), Child Health Task Force (CHTF), 
International Association of Public Health Logisticians (IAPHL), the Community Health Community of Practice, etc. were also approached to identify contact persons.

\subsection{Inclusion and Exclusion Criteria}

In this study, we defined CHWs as 'workers based in communities (i.e., conducting outreach from their homes and beyond primary health care facilities), providing health services at a community level, who are either paid or volunteer'.

56 definitions of shortage and stock-out have been used in published and grey literature. ${ }^{[]]}$For the purpose of this study, we defined stock-out as: "The complete absence of a required drug at a storage point or delivery point for at least one day." A study was included if it contained a) information on the number or percentage of $\mathrm{CHWs}$ or HFs stocked-out, or b) reasons for stock-outs at these levels. The quality of the included studies was assessed by grading, based on the criteria like, geographical coverage, sample size, information on the stock outs, mention of reasons of stock outs and the type of information source primary or secondary. Study that didn't provide the above-mentioned reasons was excluded.

Considering limited evidence available, we did not have criteria in included studies based on year of study, type of CHW, number of CHWs or HFs assessed, use of primary or secondary data, language, or geographic region. All types of commodities were included, except vaccines, as a large body of evidence exists on the status and root causes of vaccine stock-outs. [ii] [ii]

\subsection{Individual Interviews}

A purposive sample of 30 domain experts from 10 countries were identified for interviews through the network of contact persons outlined above (see Supplementary File, Appendix 3). Semi-structured interviews were conducted to validate initial findings from the studies, and to gather additional insights into the underlying root-causes of stock-outs.

\subsection{Selection and Data Extraction}

Once studies were selected, based on the inclusion criteria, relevant data were systematically extracted and tabulated in a Microsoft Excel spreadsheet. The following information was extracted from each study: (1) Study characteristics (including author, title, publication date, year of study, method of study, sample size of CHWs, sample size of HFs, geographic area, types of HFs, and list of commodities), (2) Quantification of stock-out (including number and percentage of CHWs or HFs stocked-out, percentage of commodities stocked-out, number of days commodities stocked-out at CHWs or HFs level, and frequency of stock-out at CHW or HF levels), and (3) Reasons for stock-out. We employed the mixed method ${ }^{[i v]}$, in which both qualitative and quantitative information within the same article were collected and analyzed. The qualitative data regarding the reasons for stock-out were gathered from both studies and domain expert interviews, and then were categorized under different themes, following a content analysis method. 


\section{Results}

The systematic search of both published and unpublished literature resulted in a total of 1192 records (see Supplementary File, Appendix 2). After the deletion of duplicates, 752 studies were screened in abstracts and titles, resulting in 101 studies. After full-text screening of the 101 studies, 48 studies (including 3 databases) were included in the review; the remaining 53 studies were excluded as illustrated in Figure 1. The included 48 studies contained quantified information on stock-out rate at $\mathrm{CHW}$ and/or HF levels and/or reasons for stock-outs (see Appendix 4). The quality of the included studies were analysed

\subsection{Analysis of Stock-out Rates}

We computed descriptive statistics of stock-out rates among CHWs and HFs, based on the numbers reported by various studies (See Table 1. Also see Supplementary File, Appendix 5 for the extracted values). Out of 48 included studies, 46 studies (including 3 databases) stated the number or percentage of CHWs and/or HFs stocked-out. Data analysis contained reported data from the following 19 SubSaharan African (SSA) countries.

Countries: Benin, Burkina Faso, Cameroon, Democratic Republic of Congo, Ethiopia, Kenya, Liberia, Madagascar, Malawi, Mali, Mozambique, Niger, Nigeria, Rwanda, Senegal, Sierra Leone, Tanzania, Uganda and Zambia.

We conducted a hypothesis test to understand if a statistical link existed between the CHW and HF stockout rates. The resulting P-value $[(0.0734)>0.05]$ indicated there was no significant difference in the stockout rates between $\mathrm{CHWs}$ and HFs (see Supplementary File, Appendix 6).

\subsection{Percentage of Medicines Stocked-out and Stock-out Duration}

Out of the included studies, only seven studies documented the percentage of managed commodities stocked-out among CHWs or HFs. The average percentage of managed commodities stocked-out ranged from $8.4 \%$ to $58 \%$. Managed commodities varied by country and included products for iCCM and reproductive health.

Reporting on durations of stock-outs lacked standardization across studies (see Table 2). Studies reported differing periods for stock-outs. This variation is to be expected as the period reported depends on the original data collection method (e.g. through the logistics management information system (LMIS), or review of documents, or through survey/observation on the day of visit).

\subsection{Reasons for stock-outs}

In this review, a bottleneck is defined as "an obstacle that impedes the availability of essential and program health commodities at the CHWs level".

28 of the 48 studies listed reasons for stock-outs. Semi-structured interviews with domain experts were conducted from October 2018 to December 2018 to gather information on CHW stock-out causes, to 
identify bottlenecks in SC systems, and to verify findings from the studies. Information regarding reasons for stock-out were extracted and categorized into different themes. The themes were coded to perform a frequency count. The following SC bottlenecks were mentioned most frequently (Please see Chart 1).

\subsubsection{Financial Issues}

Budgetary constraint is one of the major barriers to efficient SC functioning, ultimately leading to stockout of community-level commodities. Reviewed studies ${ }^{[i][i]}$ and domain experts also specified that inadequate finances for procurement led to national and regional level stock-outs, which reduce community-level stock availability. Lack of financial resources to procure the entire package of iCCM medicines, particularly non-malaria commodities, was a commonly reported problem. Budgetary constraints also hinder distribution, transportation, supervision, and implementing improved data systems. No details were available on whether financial constraints were due to the budget development process, allocation of funds, funding flows to subnational level, budget utilization, or a combination of all -highlighting the need for more information.

\subsubsection{Transportation}

CHWs typically work in rural locations, with difficult terrains. Commuting to resupply points is usually arduous due to poor roads, limited public transportation, or lack of transportation fund. The majority of CHWs in countries, such as Rwanda and Ethiopia ${ }^{\text {[iii] }}$, travel by foot for resupply. Lack of funding for transportation to collect supplies from HFs is an impediment limiting product availability. Furthermore, trips to the HF are often linked with monthly supervisor meetings at the HF, which become challenging to hold, for the same reasons.

\subsubsection{Data Related Issues}

Lack of information systems to capture community-level data, lack of data visibility, and lack of data reporting procedures and tools result in poor inclusion of $\mathrm{CHW}$ supply requirements in resupply calculations at all levels. In some cases, MNCH medicines are not even included in the national LMIS. According to some domain experts, several existing systems do not allow for the disaggregation of stock information between $\mathrm{CHW}$ and HF levels. There are exceptions: the cStock system in Malawi provides stock data at the community-level. ${ }^{\text {[iv] }}$ Good SC data is vital to understand consumption patterns, to inform resupply, to improve quantification and management of products.

At HF and CHW levels, poor data collection and use makes it difficult to accurately estimate needs, which are due to lack of capacity among CHWs and poor supervision. Trainings on reporting and data collection processes are limited, as per domain experts, resulting in sparse and low-quality data in those countries that capture data from $\mathrm{CHWs.}$

\subsubsection{Human Resource Issues}


Human resource challenges include: 1) lack of adequate resources, 2) inadequate staff trainings, and 3) improper remuneration. Inadequate training and limited resources hamper proper data collection and utilization, which often leads to poor demand-forecasting. Although, CHWs have been found to follow procedures and perform simple tasks correctly, given sufficient orientation and supervision, most CHWs are either inadequately paid or unpaid, resulting in low motivation. In countries such as Rwanda, more than one-third of $\mathrm{CHWs}$ have mentioned remuneration as the major constraint in managing health products. $^{[\mathrm{l}]}$

\subsubsection{Stock Management and Storage}

Limited ability of CHWs and HFs to manage stock efficiently, either due to limited knowledge of stock management practices, or inadequate or improper storage space, also leads to $\mathrm{CHW}$ stock-outs. The issue of inadequate storage space is not only confined to $\mathrm{CHWs}$, who typically store supplies in a box in their home but is more relevant for HFs and regional warehouses. Absence of basic stock management practices and poor storage conditions can affect the quality of medicines or lead to expired or overstocked commodities.

\subsubsection{Poor Forecasting}

Poor quantification at the national level leads to inadequate stock levels, which impact CHW stock-outs. In some countries, $\mathrm{CHW}$ requirements are not included in the national forecast. For example, while the cStock system tracks consumption and resupply of $\mathrm{CHWs}$, this information is not considered during the annual national-level quantification process.

\subsubsection{Issues at Resupply Points}

Inadequate supplies at resupply points, absence of or unclear resupply procedures and policies also result in stock-outs. When HFs don't receive enough supplies for their own consumption, they prioritize their needs over $\mathrm{CHWs}$, resulting in $\mathrm{CHWs}$ stock-out. ${ }^{\text {[vi] }}$ Detailed explanations for inadequate supplies at resupply points were not mentioned in the reviewed studies.

\subsubsection{Supervision}

Effective management of inventory and supply orders require regular supervision. Budgetary constraints often lead to inadequate supervision, due to lack of human resources, capacity building activities, trainings and/or transportation to conduct supervision. Lack of clear guidance also leads to irregular supervisory activities.

\subsubsection{Coordination}

Communication gaps and weak coordination across SC levels are also the reasons for stock-outs and can often lead to transparency and accountability issues. These issues limit CHWs from knowing whether or when products are available for resupply. ${ }^{[\text {[ii] }}$ In some countries, donor-funded organizations 
facilitate coordination among SC levels. In certain donor-funded projects, CHWs are restocked during monthly meetings ${ }^{[i]}$, which are used as a platform to enhance coordination and communication.

\subsubsection{Priority/Recognition of CHWs}

Some studies and domain experts indicated that $\mathrm{CHWs}$ are not fully recognized as a unit of the formal health system (integrated in the national health system). HFs are seen as the "last mile", therefore, CHW stock is, in some cases, viewed as HF stock, rather than separately. Poor recognition of and attitudes towards $\mathrm{CHWs}$ from formal health workers, along with the lack of formal policies on the products that CHWs are permitted to distribute, are major challenges in task shifting, despite the fact that some CHWs are experienced and effectively lead medicine distribution. ${ }^{[x]}$

\subsubsection{High Consumption and Distribution}

Inability to stock adequately during increased demand due to recurrence of epidemics and/or utilization of commodities, also contributes to CHW stock-outs, especially if long time lags exist between the consumption, reporting, and resupply. Varying capacity in distribution at HFs and higher levels and inaccurately captured consumption data at community-level compound this issue. Improper distribution could result in a surplus of commodities at a central level but stock-out at a community-level.

\section{Discussion}

While gathering and analyzing data for stock-out of commodities at $\mathrm{CHW}$, we encountered the following challenges:

\subsubsection{Limited Study and Inadequate Data}

Despite extensive efforts to find pertinent information, we encountered issues: few studies on CHW product availability have been conducted, the available data on stock-out frequency and underlying causes were poorly documented, and, in many cases, data were inadequate.

Based on our data analysis, most studies have focused primarily on SSA countries. Despite the fact that CHW programs in South Asia and Latin America regions have scored impressive health and social gains ${ }^{[i]}$, the product availability data from these regions are almost non-existent. Thus, the presented analysis focuses on the SSA region.

\subsubsection{Inconsistent Reporting Metrics}

Different, non-standardized metrics have been used to report stock availability for community health commodities. Compiling results from various studies is difficult due to such inconsistencies.

Standardized benchmarking is, therefore, advised to measure the extent and the causes of such stockouts. Encouraging the use of an existing, standardized set of metrics ${ }^{[\text {[i] }}$ to report on the stock-out of essential and program health commodities across countries is strongly recommended. 
Based on health situations and requirement, different countries have their own lists of essential program commodities that $\mathrm{CHWs}$ are allowed to distribute. The reviewed studies were not consistent in terms of reporting such commodities and their stock availability. For example, in some studies the aggregate stock-out percentage of all essential medicines was reported without mentioning the included product types, while in other studies, the stock-out rate of individual medicines was reported.

There is also inconsistency in reported period of stock-outs, which made it difficult to compute or to compare the exact duration. Although some studies mentioned a high frequency of stock-outs, no attempt was made to quantify such frequencies. Information about the total stock-out days along with the frequency are important to understand the extent and impact of stock-outs. These data sparsity and data quality issues impeded us from performing additional informative analyses, such as longitudinal analysis, temporal analysis, and intra/inter-country comparisons. A standard criterion for duration in the definition of a reported stock-out is recommended for future studies.

\subsection{Magnitude and Reasons for Stock-outs at CHWs level}

Stock-outs present a significant issue for quality of care. This paper's results show that almost half [48.09\%, $\mathrm{Cl}$ 95\%: 39.28-56.90] of CHWs experience stock-outs of essential program commodities. With the resulting P-value (0.0734) computed for the hypothesis test examining a significant difference between stock-out rates at $\mathrm{CHWs}$ and HFs levels, we cannot reject the null hypothesis. Based on limited data, it seems that $\mathrm{CHWs}$ and HFs experience stock-outs at similar rates, which is not unsurprising as HFs supply the CHWs in the majority of cases.

The most frequently cited reason for stock-outs at the community-level is a lack of financial resources, followed by other issues. Financial challenge also leads to national-level stock-outs, particularly of nonmalaria iCCM commodities. Besides, issues on transportation, data, human resource, and stockmanagement and storage are significant bottlenecks hindering the ability of CHWs to save lives in LMICs. Given the fact that investment in $\mathrm{CHWs}$ is essential to achieve universal health coverage and can result in

ten-fold economic returns on investment in $S \mathrm{SA}^{[\text {[iii] }}$, reducing these bottlenecks to promote community health is urgently required.

\subsection{Strengths and Limitations}

We conducted a systematic literature search that included both published and unpublished articles. A number of professional health platforms were utilized to access unpublished literature. We also attempted to gather expert views via personal communications from different organizations working in community health.

Although we employed a thorough approach, it is possible that some publications could have been unintentionally omitted in our review. For example, our query might have missed a number of titles that are used to refer to $\mathrm{CHWs}$. The included studies have used various reporting metrics to present stock-outs 
at the $\mathrm{CHW}$ and HF levels. Because of such inconsistencies, the computed stock-out statistics may not be truly representative.

\section{Conclusions}

We attempted to document the extent of community-level stock-out of essential and program commodities for MNCH in LMICs, and to understand the underlying bottlenecks that lead to such stockouts. The result shows that stock-out of health commodities is a major issue affecting almost half of CHWs, thus impacting service delivery and eroding the community's trust in the health system.

Stock-outs occur not only at the CHW level but also at higher SC levels. Many bottlenecks that contribute to stock-outs at all the levels of the SC system were identified, through reviewed studies and expert interviews. These were primarily related to problems in financing, data, and capacity to manage stock at $\mathrm{HF}$ and $\mathrm{CHW}$ levels. Adding the $\mathrm{CHW}$ level as an extension to the SC places additional strain on what are often weak supply chains; this structure compounds the identified problems. It is recommended to assess whether the SC is robust enough to support iCCM. Several studies have pointed out that more direct SC model reduces the duration and frequency of stock outs compared to conventional three-level SC system[i]. A system strengthening approach is required to reduce stock-outs at the community-level. Sparse data and inconsistent reporting metrics posed a significant challenge for analyzing results from existing studies and resulted in high variance in the findings. The sparse data on product availability among CHWs warrants additional future studies. Similarly, a set of standard metrics is recommended to measure the rate, period, and frequency of stock-outs in future.

\section{Declarations}

\section{- Ethics approval and consent to participate}

Not Applicable

\section{- Consent for publication}

Not Applicable

\section{- Availability of data and material}

All data generated or analyzed during this study are included in its supplementary information file.

\section{- Competing interests}

The authors declare that they have no competing interests

\section{- Funding}


The study is funded by UNICEF, Health Section, NYHQ. JB's contribution was funded under the US Agency for International Development (USAID) Medicines, Technologies, and Pharmaceutical Services (MTaPS) project. The funders had no role in study design, data collection and analysis, decision to publish, or preparation of the manuscript. Its contents are solely the responsibility of the authors and do not necessarily represent the official views of UNICEF Health Section NYHQ or any other organizations of which the officials are involved.

\section{- Authors' contributions}

AP collected, analyzed and interpreted the data on CHW and HF stock-out and was a major contributor in writing the manuscript, EB assisted in collecting the data and in editing the manuscript, HSD and JB contributed to the study design and presentation of the findings and reviewed drafts of the article, $\mathrm{BS}, \mathrm{HH}$ and MB helped in editing the manuscripts. All authors read and approved the final manuscript.

\section{- Acknowledgements}

We thank all the experts that helped us by providing insights on the issues of Community Health Workers and provided us with the database to analyze information. Special thanks to Ms. Hannah Sarah Dini, Program Specialist, UNICEF for coordinating the research activities by connecting to the potential professionals and organizations for collecting data.

\section{Abbreviations}

$\begin{array}{ll}\text { CHTF } & \text { Child Health Task Force } \\ \text { CHW } & \text { Community Health Worker } \\ \text { HF } & \text { health facility } \\ \text { IAPHL } & \text { International Association of Public Health Logisticians } \\ \text { iCCM } & \text { Integrated Community Case Management } \\ \text { ISG } & \text { Interagency Supply Chain Group } \\ \text { JSI } & \text { John Snow, Inc. } \\ \text { LMICs } & \text { Low- and Middle-Income Countries } \\ \text { LMIS } & \text { Logistics Management Information System } \\ \text { MNCH } & \text { Maternal, Newborn, and Child Health } \\ \text { MSH } & \text { Management Sciences for Health } \\ \text { SC } & \text { supply chain }\end{array}$

SC4CCM Improving Supply Chains for Community Case Management of Pneumonia and Other Common Diseases of Childhood

SHWG Systems for Health Working Group

USAID United States Agency for International Development

WHO World Health Organization

\section{References}

1. U.S. Department of Health and Human Services Health Resources and Services Administration Bureau of Health Professions. Community Health Worker National Workforce Study: An Annotated 


\section{Bibliography. March 2007}

2. Diana F., Maryse K., Korrie de K., and Estelle Q. Community Health Workers and Universal Health Coverage. Knowledge gaps and a need based Global Research agenda by 2015. 2013.

3. UNICEF. Access to healthcare through community health workers in East and Southern Africa. Maternal, Newborn and Child Health Working Paper UNICEF Health Section, Program Division. 2014.

4. Ibid

5. World Health Organisation and Global Health Workforce Alliance. Global Experience of Community Health Workers for Delivery of Health-Related Millennium Development Goals: A Systematic Review, Country Case Studies, and Recommendations for Integration into National Health Systems. 2010.

6. Ballard M, Madore A, Johnson A, et al. Concept Note: Community Health Workers. Harvard Business Publishing. 2018.

7. Lassi ZS, Haider BA, Bhutta ZA. Community-based intervention packages for reducing maternal and neonatal morbidity and mortality and improving neonatal outcomes. Cochrane Database of Systematic Reviews; 2010 Issue 11. Art. No.: CD007754. DOI: 10.1002/14651858.CD007754.pub2

8. Amouzou, A., Morris, S., Moulton, L. H., \& Mukanga, D. Assessing the impact of integrated community case management (iCCM) programs on child mortality: Review of early results and lessons learned in sub-Saharan Africa. Journal of global health; 2014 4(2), 020411.

9. Kanté AM, Exavery A, Jackson EF, et al. The impact of paid community health worker deployment on child survival: the connect randomized cluster trial in rural Tanzania. BMC Health Serv Res. 2019;19(1):492. Published 2019 Jul 16. doi:10.1186/s12913-019-4203-1

10. Dahn B. et. al. Strengthening Primary Health Care through Community Health Workers: Investment Case and Financing Recommendations. 2015; Ballard, M. and P. Montgomery, Systematic review of interventions for improving the performance of community health workers in low-income and middleincome countries. BMJ Open, 2017. 7(10): p. e014216.

11. Baker K, Akasiima M, Wharton-Smith A, et al. Performance, Acceptability, and Usability of Respiratory Rate Timers and Pulse Oximeters When Used by Frontline Health Workers to Detect Symptoms of Pneumonia in Sub-Saharan Africa and Southeast Asia: Protocol for a Two-Phase, Multisite, MixedMethods Trial. JMIR Res Protoc. 2018;7(10):e10191. Published 2018 Oct 25. doi:10.2196/10191

12. Zulu, J.M., et al., Integrating national community-based health worker programmes into health systems: a systematic review identifying lessons learned from low- and middle-income countries. BMC Public Health\, 2014. 14\: p. 987\.; Bhutta, Z.A., et al., Global experience of community health workers for delivery of health-related millennium development goals: a systematic review, country case studies. Global Health Workforce Alliance, 2010. 1: p. 249-61.; Jaskiewicz, W. and Tulenko, K., Increasing community health worker productivity and effectiveness: a review of the influence of the work environment. Human Resources for Health, 2012. 10(1): p. 38.

13. Gopalan SS, Mohanty S, Das A. Assessing community health workers' performance motivation: a mixed-methods approach on India's Accredited Social Health Activists (ASHA) programme. BMJ Open 2012;2:e001557. doi:10.1136/bmjopen-2012-001557 
14. Cometto, G., et al., Health policy and system support to optimise community health worker programmes: an abridged WHO guideline. The Lancet Global Health, 2018. 6(12): p. e1397-e1404; Ballard, M. and R. Schwarz, Employing practitioner expertise in optimizing community healthcare systems. Healthcare, 2018.

15. WHO. Meeting Report: Technical Definitions of Shortages and Stockouts of Medicines and Vaccines. 2006.

16. Lydon, P., Schreiber, B., Gasca, A., Dumolard, L., Urfer, D. and K. Senouci,

17. Vaccine stock-outs around the world: Are essential vaccines always available when needed?, Vaccine. 2017, 35(17), 2121-2126.

18. WHO. Global Vaccine Action Plan Monitoring, Evaluation \& Accountability Secretariat Annual Report. 2018.

19. Kaur M. Application of Mixed Method Approach in Public Health Research. Indian J Community Med. 2016;41(2):93-7.

20. Namazzi G, Waiswa P, Nakakeeto $M$, et al. Strengthening health facilities for maternal and newborn care: experiences from rural eastern Uganda. Glob Health Action. 2015;8:24271. Published 2015 Mar 31. doi:10.3402/gha.v8.24271

21. World Health Organisation. Summative evaluation of the WHO Rapid Access Expansion Initiative. 2018

22. Chandani, Y., Noel, M., Pomeroy, A., Andersson, S., Pahl, M. K., \& Williams, T. (2012) Factors Affecting Availability of Essential Medicines among Community Health Workers in Ethiopia, Malawi, and Rwanda: Solving the Last Mile Puzzle. The American Journal of Tropical Medicine and Hygiene, 87(5 Suppl), 120-126. http://doi.org/10.4269/ajtmh.2012.11-0781

23. World Health Organisation. Better access to life-saving medicines through interactive SMS in Malawi: JSI's cStock.2014

24. Chandani, Y., Andersson, S., Heaton, A., Noel, M., Shieshia, M., Mwirotsi, A., Krudwig, K., Nsona, H., Felling, B. Making products available among community health workers: Evidence for improving community health supply chains from Ethiopia, Malawi, and Rwanda. Journal of global health, 2014. 4(2), 020405.

25. Information extracted from interviews with domain-experts.

26. WHO. Summative evaluation of the WHO Rapid Access Expansion Initiative Volume 3: Country Briefs. 2018.

27. Information extracted from interviews with domain-experts.

28. Ibid

29. Ibid

30. World Health Organisation and Global Health Workforce Alliance. Global Experience of Community Health Workers for Delivery of Health-Related Millennium Development Goals: A Systematic Review, Country Case Studies, and Recommendations for Integration into National Health Systems. 2010. 
31. USAID. Indicator Guide Monitoring and Evaluating Integrated Community Case Management. July 2013

32. Dahn B. et. al. Strengthening Primary Health Care through Community Health Workers: Investment Case and Financing Recommendations. 2015.

\section{Tables}

Table 1: Statistics on the magnitude of stock-out of at least one medicine among CHWs and HFs

\begin{tabular}{|l|c|c|}
\hline Statistics & CHWs & HFs \\
\hline AVERAGE (\%) & $\mathbf{4 8 . 0 9}$ & $\mathbf{5 4 . 7 6}$ \\
\hline Range (\%) & $4.00-91.70$ & $5.00-100.00$ \\
\hline STDEV & 25.02 & 27.46 \\
\hline Median (\%) & 41 & 48 \\
\hline Variance & 626.05 & 753.83 \\
\hline Sample size* & 31 studies & 23 studies \\
\hline 95\% Confidence Interval & [39.28 - 56.90] & [43.54 - 65.98] \\
\hline
\end{tabular}

* Out of the total included, 26 studies had data on CHW stock-out, 4 studies had data on both CHW and HF stock-outs, and 19 studies had data on HF stock-out rates. Some studies had multi-country information, therefore the number of data points and studies do not match.

Table 2: Examples of stocked-out period as reported by various studies 


\begin{tabular}{|l|l|l|l|}
\hline \multicolumn{1}{|c|}{ Studies** } & Country & Geographical representation & $\begin{array}{l}\text { Reported Stock-out } \\
\text { period for CHWs or } \\
\text { HFs }\end{array}$ \\
\hline $\begin{array}{l}\text { Lufesi M. } \\
\text { et al., } \\
2007\end{array}$ & Malawi & Lilongwe District & 6 months on average \\
\hline $\begin{array}{l}\text { Doherty T. } \\
\text { et al., } \\
2014\end{array}$ & Ethiopia & $\begin{array}{l}\text { 239 woredas in 26 zones in 5 regions } \\
\text { (Tigray, Amhara, SNNP, Oromia and } \\
\text { Benishangul-Gumuz) }\end{array}$ & $3-4$ months \\
\hline $\begin{array}{l}\text { Gils T. et } \\
\text { al., 2018 }\end{array}$ & DRC & Kinshasa & 40 days on average \\
\hline $\begin{array}{l}\text { Smith S. } \\
\text { et al., } \\
2013\end{array}$ & Madagascar & 30 districts out of 114 & $\begin{array}{l}\text { 2-3 times per year, } \\
\text { lasting one month or } \\
\text { longer }\end{array}$ \\
\hline $\begin{array}{l}\text { Doherty T. } \\
\text { et al., } \\
2014\end{array}$ & Ghana & 3 northern regions and the Central Region & More than 1 week \\
\hline $\begin{array}{l}\text { Munos M. } \\
\text { et al., } \\
2016\end{array}$ & $\begin{array}{l}\text { Burkina } \\
\text { Faso }\end{array}$ & $\begin{array}{l}\text { North and Central North Rapid Scale-Up } \\
\text { districts }\end{array}$ & 7 days or more \\
\hline $\begin{array}{l}\text { WHO, } \\
2018\end{array}$ & Niger & 4 districts in 2 regions (Dosso and Tahuoa) & $\begin{array}{l}\text { At least 7 days in the } \\
\text { past month }\end{array}$ \\
\hline $\begin{array}{l}\text { Miller } \\
\text { N.P. et al., } \\
2014\end{array}$ & Ethiopia & $\begin{array}{l}\text { Rural areas of Jimma and West Hararghe } \\
\text { Zones of Oromia Region }\end{array}$ & Day of visit \\
\hline
\end{tabular}

** Lufesi, N. N., Andrew, M., \& Aursnes, I. Deficient supplies of drugs for life threatening diseases in an African community. 2007, BMC health services research, 7, 86.

doi:10.1186/1472-6963-7-86; Doherty T., Loveday M., Nsibande D., Daniels K., Daviaud E., Kerber K., Zembe W., Leon N., Kinney M. Report on the Summative External Evaluation of the Catalytic Initiative (CI)/ Integrated Health Systems Strengthening (IHSS) Programme in Ethiopia. 2016; Gils, T., Bossard, C., Verdonck, K., Owiti, P., Casteels, I., Mashako, M., Van Cutsem, G., Ellman, T. Stockouts of HIV commodities in public health facilities in Kinshasa: Barriers to end HIV. PloS one. 2018, 13(1), e0191294. doi:10.1371/journal.pone.0191294' Smith S. et. al. (2013) Community Health Volunteer Program Functionality and Performance in Madagascar: A Synthesis of Qualitative and Quantitative Assessments; Doherty T, Daniels K, Daviaud E, Kinney M, Ngandu N. Report on the Summative External Evaluation of the Catalytic Initiative (CI)/ Integrated Health Systems Strengthening (IHSS) Programme in Ghana. Cape Town: South African Medical Research Council, University of the Western Cape and Save the Children. 2014; Munos, M., Guiella, G., Roberton, T., Maïga, A., Tiendrebeogo, A., Tam, Y., Bryce, J., Baya, B. Independent Evaluation of the Rapid Scale-Up Program to Reduce UnderFive Mortality in Burkina Faso. The American journal of tropical medicine and hygiene. 2016, 94(3), 584-95; WHO. Summative evaluation of the WHO Rapid Access Expansion Initiative Niger. 2018; Miller, N. P., Amouzou, A., Tafesse, M., Hazel, E., Legesse, H., Degefie, T., Victora, C. G., Black, R. E. Bryce, J. Integrated community case management of childhood 
Illness in Ethiopia: implementation strength and quality of care. The American journal of tropical medicine and hygiene, 2014, 91(2), 424-34.

\section{Figures}

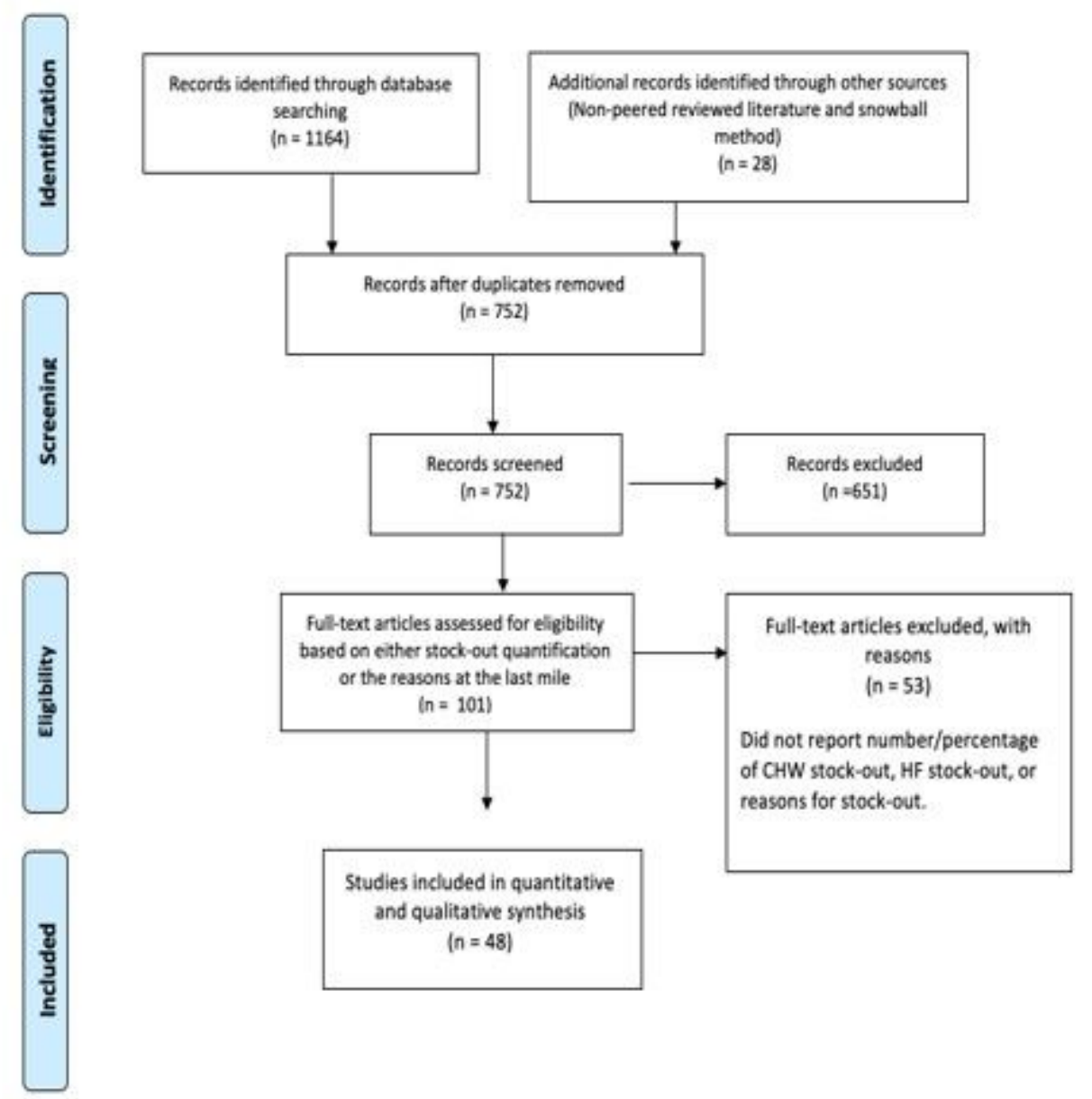

\section{Figure 1}

Flow diagram

\section{Supplementary Files}

This is a list of supplementary files associated with this preprint. Click to download.

- PRISMA2009checklist.doc

- SupplementaryFileonStockoutsandbottlenecks.docx

- Chart.docx 